\title{
The pattern and prevalence of vertebral artery injury in patients with cervical spine fractures
}

\author{
F Ismail, ${ }^{1}$ FCRad (D) SA, MMed Rad (D); S Motsitsi, ${ }^{2}$ MMed (Orth), FCS; N Khan, ${ }^{3}$ MB BS, FC Rad; \\ I Fabris-Rotelli, ${ }^{4}$ BSC (Hons), MSc, PhD
}

\author{
${ }^{1}$ Department of Radiology, University of Pretoria, Pretoria, South Africa \\ ${ }^{2}$ Department of Orthopaedic Surgery, Kalafong Hospital, Pretoria, South Africa \\ ${ }^{3}$ Department of Radiology, Kalafong Hospital, Pretoria, South Africa \\ ${ }^{4}$ Department of Statistics, University of Pretoria, Pretoria, South Africa
}

Corresponding author: F Ismail (drfismail@yahoo.com)

\begin{abstract}
Aim. It is not uncommon for vertebral artery injury to occur when there are fractures through the transverse foraminae of the first to the sixth vertebral bodies. Other important risk factors for vertebral artery injury include facet joint dislocations and fractures of the first to the third cervical vertebral bodies. The aim of this study was to determine the pattern and prevalence of vertebral artery injury on CT angiography (CTA) in patients with cervical spine fractures.

Method. A retrospective review of patients who had undergone CTA of the vertebral arteries was undertaken. Reports were reviewed to determine which patients met the inclusion criteria of having had both cervical spine fractures and CTA of the vertebral arteries. Images of patients who met the inclusion criteria were analysed by a radiologist.

Results. The prevalence of vertebral artery injury was 33\%. Four out of the 11 patients who had vertebral artery injury had post-traumatic spasm of the artery, with associated thrombosis or occlusion of the vessel. In terms of blunt carotid vertebral injury (BCVI) grading, most of the patients sustained grade IV injuries. Four patients who had vertebral artery injury had fractures of the upper cervical vertebrae, i.e. C1 - C3. Fifteen transverse process fractures were associated with vertebral artery injury. No vertebral artery injury was detected in patients who had facet joint subluxations. Conclusion. Patients with transverse process fractures of the cervical spine and upper cervical vertebral body fractures should undergo CTA to exclude vertebral artery injury.
\end{abstract}

\section{S Afr J Rad 2013;17(2):52-55. DOI:10.7196/SAJR.772}

Vertebral artery injuries are rare, with an incidence of $0.1-1.0 \%$, if all patients admitted with blunt head trauma are considered. ${ }^{[1]}$ It is not unusual for vertebral artery injury to occur when there are fractures through the transverse foraminae of the first to the sixth vertebrae. Woodring et al. ${ }^{[2]}$ found the incidence of vertebral artery injury to be $78 \%$ in patients who had fractures through the transverse foraminae. Other important risk factors for vertebral artery injury include facet joint dislocations and fractures of the first to the third cervical vertebrae. ${ }^{[3,4]}$

Injury to the vertebral arteries can result in permanent brain injury via ischaemia to the posterior circulation territory of the brain. Vertebral-basilar insufficiency may occur if either both vertebral arteries or the dominant vertebral artery is injured. Other sequelae include downstream embolisation of a thrombus, anterior spinal artery compromise causing spinal cord ischaemia, and occlusion of blood flow to the posterior inferior cerebellar artery (PICA), causing lateral medullary syndrome. ${ }^{[5]}$

Computed tomography angiography (CTA) is becoming the study of choice (rather than digital subtraction angiography (DSA)) for imaging the vertebral arteries in trauma. CTA is easily accessible, has no risk associated with an interventional procedure, and is more quickly and easily obtained in centres where both CTA and DSA are available. In addition to visualisation of the vertebral arteries, CTA also allows visualisation of the carotid arteries. At Kalafong Hospital in Pretoria, CTA is routinely used as a screening tool to exclude vertebral artery injury in all patients who have fractures involving the transverse foraminae of the cervical spine, those with facet joint dislocations, and those with fractures involving the first to the third cervical vertebrae. The aim of this study was to determine the pattern and prevalence of vertebral artery injury using CTA in patients with cervical spine fractures, and to compare these findings with those from the international literature.

\section{Methods}

A retrospective review of data from patients who had undergone CTA of the vertebral arteries was undertaken at Kalafong Hospital, a secondary hospital in western Tshwane in Gauteng Province. Ethical clearance for the study was obtained from the Main Ethics Committee of the University of Pretoria.

CTA was performed on a Philips multi-detector CT scanner (Brilliance 16, Netherlands; Serial Number 5067). Routine CTA protocols of the neck are titled 'CT neck angiogram', 'CT cervical spine angiogram' and 'CT carotid angiogram. The CT scan database was searched according to these protocol titles. The CT database also has a list of all patients who have been scanned, as well as the type 
of scan that was performed. Reports were reviewed to determine which patients met the inclusion criteria of having had cervical spine fractures and/or subluxations, as well as CTA of the vertebral arteries. All CT scans and reports were checked by a consultant radiologist. Images of all patients are archived on erasable optical discs (EODs). Images of patients who met the inclusion criteria were accessed from the EODs and analysed by a radiologist. The standardised method of performing CTA of the vertebral arteries uses a bolus tracking technique with the region of interest (ROI) placed in the aortic arch. One hundred $\mathrm{ml}$ of iodinated contrast medium (Omnipaque $350 \mathrm{mg} \mathrm{I} / \mathrm{ml}$, GE Health), was administered at a flow rate of $3-4$ $\mathrm{ml} / \mathrm{s}$, followed by a normal saline bolus of $50 \mathrm{ml}$. The scan parameters were $360 \mathrm{~mA} / \mathrm{s}$ per slice, $120 \mathrm{kV}$, a slice thickness of $0.8 \mathrm{~mm}$ with slice increments of $0.4 \mathrm{~mm}$, a field of view (FOV) of $220 \mathrm{~mm}$, and pitch of 0.438. Scans extended from the aortic arch to the posterior fossa of the brain. Multiplanar reconstruction in coronal and sagittal planes in bone window (using a window of 2000 Hounsfield units (HU) and centre level of $800 \mathrm{HU}$ ) was used to assess for fractures. Maximal intensity projection (MIP) reconstructions in axial, coronal and sagittal planes, at a slice thickness of $3-4 \mathrm{~mm}$ (varied between the scans), window level of approximately $745 \mathrm{HU}$ and centre of $228 \mathrm{HU}$ were used to assess the vertebral arteries.

The age, gender, mechanism of injury, fracture level of the transverse processes, and description of the vertebral artery injuries were documented. Fractures of the vertebral bodies and the presence of subluxations were also documented. Transverse processes included the transverse foraminae and anterior and posterior tubercles. Transverse process fractures were numbered $\mathrm{C} 1-\mathrm{C} 7$ and separated right from left, and the level of vertebral artery injury was marked V1 - V7, corresponding to the level of cervical spine vertebrae.

The blunt carotid and vertebral artery injuries (BCVI) grading system was used to grade the extent of vertebral artery injuries. ${ }^{[6]}$ The BCVI grading system defines vessel injury as follows: Grade I is the presence of luminal irregularity or dissection with an intra-luminal haematoma occluding less than $25 \%$ of the lumen. Grade II is similar to grade I, except that $25 \%$ or more of the vessel lumen is occluded. Grade III lesions are due to pseudo-aneurysms, grade IV lesions are due to total occlusion of the vessel and transection of the vessel are grade $\mathrm{V}$ injuries.

Post-traumatic spasm and congenital hypoplasia both have a similar imaging appearance, which is diffuse narrowing of the affected vertebral artery. The distinction between post-traumatic spasm of the vertebral artery and congenital hypoplasia is determined by comparing the anterior-posterior (AP) diameter of the transverse foramina on the right and the left. The level that was chosen for measurement was individualised for each patient, at a point where there was no injury to the vessel and no vertebral fracture. When the neural foraminae were of similar size and the vessel calibre was disproportionately smaller, the affected vessel was considered to be in spasm.

Stenosis of the vessel lumen was defined as a local or general decrease in calibre owing to the presence of an intraluminal thrombus. The degree of stenosis was calculated by measuring the narrowest diameter of the flow channel and expressing this as a percentage of the diameter of the vessel, including the thrombus, on axial maximum intensity projection (MIP) images (corrected for vessel tortuosity).

All available patient data were used. Data were captured on a paper data collection sheet and later transferred to a Microsoft Excel spreadsheet. Only descriptive analysis of the data was undertaken, owing to small patient numbers.

\section{Results}

Forty-two patients met the inclusion criteria. Nine patients were excluded from the initial database, as the $\mathrm{CT}$ images were not accessible from the EODs because of errors in saving the data at the time of performing the scan. The final patient sample size was 33 .

Demographic data and the mechanism of injury for each patient are summarised in Table 1. Twenty-three (70\%) patients were male, and 10 (30\%) patients were female. The median age was 35 years. Regarding the mechanism of injury, $29(88 \%)$ patients were involved in a motor vehicle accident, 2 (6\%) were assaulted, $1(3 \%)$ was involved in a pedestrianvehicle accident, and 1 (6\%) patient fell off a bicycle.

Sixty-six segmental fractures of the transverse processes were detected: 44 on the right side and 22 on the left. Twenty-six (39\%) fractures were located in the upper cervical vertebrae, i.e. C1 - C3.

Table 1. Summary of demographic data and mechanism of injury

\begin{tabular}{ll}
\hline & $\boldsymbol{n}(\%)$ \\
\hline Gender & $23(70)$ \\
Male & $10(3)$ \\
Female & \\
Age (years) & 35 \\
$\quad$ Median & $27-43$ \\
Interquartile ratio & \\
Mechanism of injury & $29(88)$ \\
Motor vehicle accident & $2(6)$ \\
Assault & $1(3)$ \\
Pedestrian/vehicle accident & $1(3)$ \\
Fell off a bicycle &
\end{tabular}

Table 2. Description of the level of vertebral artery injury in relation to the level of vertebral artery spasm

\begin{tabular}{ll}
\hline Vertebral artery spasm level & Vertebral artery injury level \\
\hline From origin to C2 & Complete occlusion from C3 to C6 \\
From origin to C7 & Eccentric thrombus compressing the lumen \\
From origin to C3 & Complete occlusion from C2 to C3 \\
From origin to C1 & No vessel injury \\
From C2 to C7 & Complete occlusion at C2
\end{tabular}


Three patients had bilateral facet joint subluxations. Two of these were located at C5 - C6 and 1 was at C2 - C3. Twenty-five (76\%) patients had unilateral transverse process fractures, and 8 (24\%) patients had bilateral fractures. Fifteen (23\%) transverse process fractures were associated with vertebral artery injury.

Eleven $(33 \%)$ patients sustained a vertebral artery injury. Five of the 11 patients had fractures of $\mathrm{C} 1-\mathrm{C} 3$ vertebral bodies. Five of the 11 had diffuse spasm and, of these, 4 had associated thrombosis or occlusion of the vessel; this is described in Table 2. Six patients sustained BCVI grade IV injury. Four patients sustained BCVI grade II injury.

Four patients who had a vertebral artery injury had fractures of the upper cervical vertebrae, i.e. C1 - C3. No vertebral artery injury was detected in patients who had facet joint subluxations.

\section{Discussion}

In this study, the prevalence of vertebral artery injury in the trauma setting was found to be $33 \%$. In a systematic review by Inamasu and Guiot, ${ }^{[3]}$ an average prevalence rate of $37.8 \%$ for vertebral artery injury was found from similar studies carried out between 1999 and 2004. Therefore our prevalence is similar to previous international studies (Pubmed and Medline search). ${ }^{[3]}$

Vertebral artery diameter is normally between $3 \mathrm{~mm}$ and $5 \mathrm{~mm}$. A hypoplastic vertebral artery is defined as having a diameter $\leq 2 \mathrm{~mm}$ and that makes little contribution to the basilar artery. The left vertebral artery is dominant in $50 \%$ of the population, the right in $25 \%$, and the arteries demonstrate equal calibre in $25 \%$ of the population. ${ }^{[7]}$ When CTA is interpreted, the distinction between a hypoplastic vertebral artery and a vertebral artery that is diffusely smaller in size, owing to post-traumatic spasm, is extremely important. To help distinguish the 2 possibilities, we compared the AP diameters of the right transverse foramina with the left transverse foramina on an axial CT slice, and compared this with the size of the vertebral artery at the same level. When the transverse foraminae were of a similar size and the vessel calibre was disproportionately smaller, the affected vessel was considered to be in spasm (Fig. 1). In this study, 4 out of the 5 patients who had diffuse spasm of the vertebral artery had associated thrombosis (1 patient at C7) or occlusion (3 patients at levels C3 - C6, C2 - C3 and at C2) (Fig. 2).

The method of measuring the AP diameter of the transverse foraminae to correlate vertebral artery size has not been proven in the literature, but was adopted for this study and proved to be a useful clue to alert the radiologist that a more sinister injury may be present.

Limitations of this study included (i) the loss of data, which affected the sample size; and (ii) the lack of current literature describing the method implemented to differentiate vertebral artery stenosis from congenital hypoplasia. Although the latter was found to be useful in this study, further research is required.

In terms of BCVI grading, 6 of the 11 patients sustained grade IV injuries, which is associated with a $44 \%$ risk of stroke and a $22 \%$ risk of mortality. ${ }^{[6]}$ Four of the 11 patients sustained grade II injuries, which is associated with an $11 \%$ risk of stroke and an $11 \%$ risk of mortality. Some studies describe a higher prevalence of grade I and II injuries, while others describe a higher prevalence of grade IV injuries. ${ }^{[8,9]}$

In 1 patient, the vertebral artery injury occurred at C7 but the fracture was at C5. It is important to evaluate all vertebral levels for fractures that may occur distant to the level of vertebral artery injury.

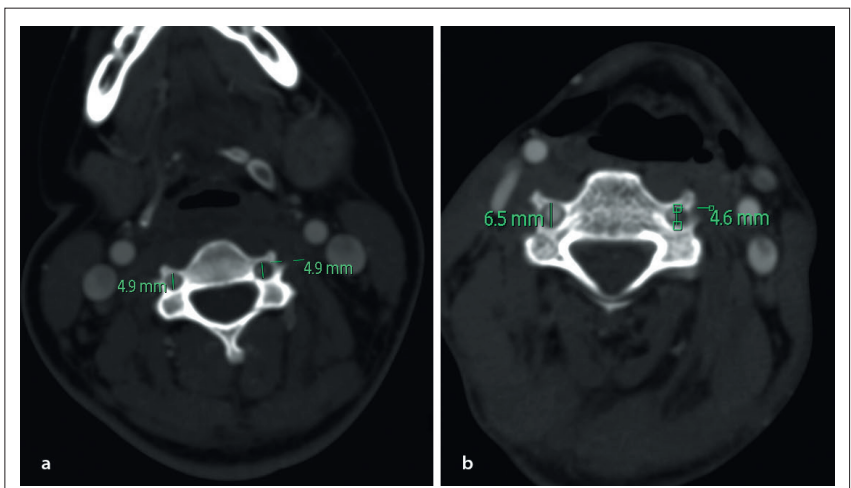

Fig. 1. Axial CTA of the vertebral arteries. In (a) the AP diameter of the transverse foraminae is equal. The left vertebral artery is much smaller than the right due to post-traumatic spasm. In (b) the AP diameter of the left transverse foramen is much smaller than the right. The left vertebral artery is proportionately smaller. This indicates a hypoplastic left vertebral artery.

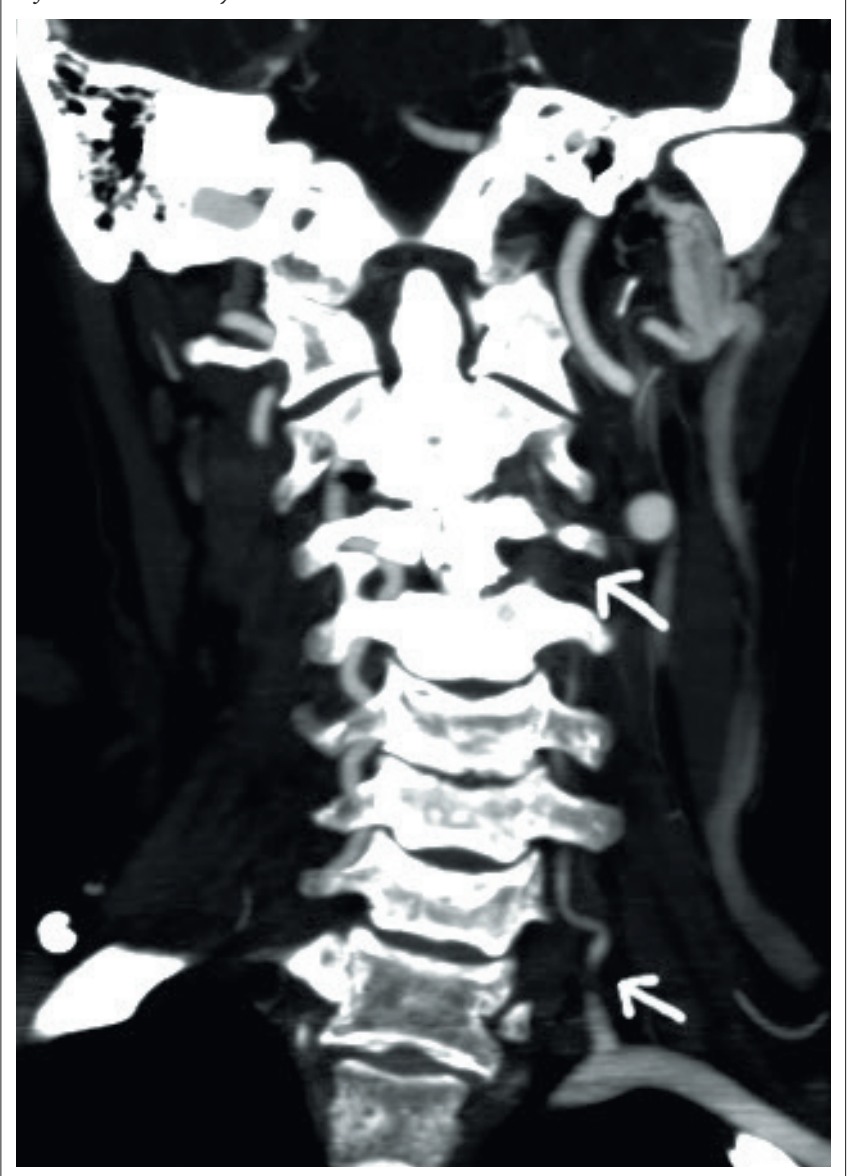

Fig. 2. Coronal MIP of a CTA of the vertebral arteries. The fracture involves the left transverse process of $C 3$. There is diffuse spasm of the left vertebral artery with associated complete occlusion of the artery at C7 and at C3 - C4 on the left (arrows).

\section{Conclusion}

Patients with transverse process fractures of the cervical spine and upper cervical vertebral body fractures should have CTA to exclude vertebral artery injury. 


\section{ORIGINAL ARTICLE}

Acknowledgements. The authors thank Mrs Johanna Masango for her outstanding assistance in image retrieval.

1. Hoit DA, Schirmer CM, Weller SJ, Lisbon A, Edlow JA, Malek AM. Angiographic detection of carotid and vertebral arterial injury in the high-energy blunt trauma patient. J Spinal Disord Tech 2008;21:259266. [http://dx.doi.org/10.1097/BSD.0b013e31814lfce8]

2. Woodring JH, Lee C, Duncan V. Transverse process fractures of the cervical vertebrae: Are they insignificant? J Trauma 1993;34:797-802. [http://dx.doi.org/10.1097/00005373-199306000-00008]

3. Inamasu J, Guiot BH. Vertebral artery injury after blunt cervical trauma: An update. Surg Neurol 2006;65:238-246. [http://dx.doi.org/10.1016/j.surneu.2005.06.043]

4. Cothren CC, Moore EE, Biffl WL. Cervical spine fracture patterns predictive of blunt vertebral artery injury. J Trauma 2003;55:811-813. [http://dx.doi.org/10.1097/01.TA.0000092700.92587.32]
5. Fassett DR, Dailey AT, Vaccaro AR. Vertebral artery injuries associated with spinal injuries: A review of the literature. J Spinal Disord Tech 2008;21:252258. [http://dx.doi.org/10.1097/BSD.0b013e3180cab162] 6. Biffl WL, Moore EE, Offner PJ, Brega KE, Franciose RJ, Burch JM. Blunt carotid arterial injuries: Implications of a new grading scale. J Trauma 1999;47:845-853. [http://dx.doi.org/10.1097/00005373199911000-00004]

7. Cloud GC, Markus HS. Diagnosis and management of vertebral artery stenosis. Q J Med 2003;96:27-34. [http://dx.doi.org/10.1093/qjmed/hcg003]

8. McKinney A, Ott F, Short J, McKinney Z, Truwit C. Angiographic frequency of blunt cerebrovascular injury in patients with carotid canal or vertebral foramen fractures on multidetector CT. Eur J Radiol 2007;62:385-393. [http://dx.doi.org/10.1016/j.ejrad.2007.01.008]

9. Biffl WL, Egglin T, Benedetto B, Gibbs F, Cioffi WG. Sixteen-slice computed tomographic angiography is a reliable non-invasive test for clinically significant blunt cerebrovascular injuries. J Trauma, Injury Infection and Critical Care 2006;60:745-752. [http://dx.doi.org/10.1097/01.ta.0000204034.94034.c4] 